\title{
Hábitos alimenticios y ecología trófica del pez Lutjanus griseus (Pisces: Lutjanidae) asociado a la vegetación sumergida en la Laguna de Términos, Campeche, México
}

\author{
Emma Guevara ${ }^{1}$, Hernán Álvarez ${ }^{2}$, Maite Mascaró ${ }^{3}$, Carlos Rosas ${ }^{3}$ \& Alberto Sánchez ${ }^{4}$ \\ 1 Universidad Autónoma del Carmen, Ave. Concordia, no. 4, Cd. del Carmen, Camp., México; \\ eguevara@pampano.unacar.mx \\ 2 Estación El Carmen, Instituto de Ciencias del Mar y Limnología, UNAM, Apdo. Postal 30, Ciudad del Carmen, Camp, \\ México. \\ 3 Laboratorio de Biología Marina Experimental, Facultad de Ciencias, UNAM, Apdo. Postal 69, Ciudad del Carmen, \\ Camp. México. \\ 4 Universidad Juárez Autónoma de Tabasco, Villahermosa 8600, Tabasco, México.
}

Recibido 05-II-2002. Corregido 12-IV-2007. Aceptado 14-V-2007.

\begin{abstract}
Feeding habits and trophic ecology of the fish Lutjanus griseus (Pisces: Lutjanidae) associated with submerged vegetation in Laguna de Términos, Campeche, Mexico. In Campeche, Mexico, the Laguna de Términos has Thalassia testudinum beds inhabited by the grey snapper, Lutjanus griseus (Linnaeus 1758). Along one year, we collected 994 individuals and 672 had food in their stomachs; we recorded number, weight, and frequency of trophic groups, and the index of relative importance. Dominant food components were Farfantepenaeus duorarum and Palemonetes octaviae, Eucinostomus gula (Cuvier 1830) and Libinia dubia. Salinity and temperature were related to the number of individuals collected and with greater fish consumption by large $L$. griseus. The trophic niche breadth index was calculated for six size-classes of fish. The smallest and the largest fish had the lowest index values, whereas medium-size snappers had higher values. This snapper has a preference for habitats with submerged vegetation. The percentage of fish with food in their stomachs was higher during dark hours, providing evidence of the nocturnal habits of this fish. Rev. Biol. Trop. 55 (3-4): 9891004. Epub 2007 December, 28.
\end{abstract}

Key words: trophic spectrum, estuarine fish, sea grasses, Lutjanus griseus, Laguna de Términos, Mexico.

Las praderas de pastos marinos revisten singular importancia ecológica ya que soportan comunidades altamente diversificadas y productivas representando una base nutricional muy importante en lo que se refiere a las redes tróficas (Mcroy y Helfferich 1977). Se ha demostrado que los depredadores ejercen una fuerte presión sobre las poblaciones de nivel trófico inferior en las comunidades que se desarrollan en fondos blandos (Peterson 1981, Jensen y Jensen 1985), de ahí que en los estudios de ecología costera el análisis de la alimentación y los hábitos alimentarios de la macrofauna son aspectos que mayor información ecológica proporcionan. La descripción de la trama trófica permite entender como se produce el flujo de la energía entre poblaciones heterogéneas de la comunidad (Rivera 1990).

En la Sonda de Campeche, ubicada en el sureste de México, se desarrollan importantes actividades pesqueras e industriales, es la segunda zona de pesca más importante de México alcanzando producciones de 50 930 toneladas anuales (SEMARNAP 2000) y sostiene la mayor producción petrolera, con el $80 \%$ de la producción nacional (Botello et al. 1992, Aguirre-León y Díaz-Ruiz 2000). La adecuada caracterización de los ecosistemas en 
los que se llevan a cabo actividades productivas permite establecer el grado de alteraciones ecológicas que se producen en los mismos debido al incremento de la actividad antropogénica (Aguilar et al. 1992a). Los estudios de la biología y ecología de especies dominantes pueden ayudar a detectar las consecuencias de la explotación comercial así como de la degradación ambiental.

La Laguna de Términos y sus sistemas fluvio-deltaicos asociados presentan una gran variedad de peces que son capturados tradicionalmente en pesquerías artesanales (AguirreLeón y Díaz-Ruiz 2000). El Cayo, en la Laguna de Términos, es un área de vegetación sumergida que presenta fondos cubiertos permanentemente por parches de pastos marinos Thalassia testudinum (Sánchez 1993). El pargo Lutjanus griseus (Linnaeus, 1758) es una especie abundante en El Cayo y considerado un consumidor de tercer orden en la áreas de pastizales de la Laguna de Términos (Yañez-Arancibia y Lara-Domínguez 1983). Si asumimos que $L$. griseus es un depredador tope, el conocimiento de sus hábitos alimenticios y su relación con los fondos de vegetación sumergida constituye una fuente de información necesaria en estudios de las comunidades establecidas en esta localidad. Además, esta información puede ser de utilidad para realizar comparaciones con otras áreas tropicales de características semejantes. El análisis del contenido estomacal de las poblaciones de L. griseus que habitan en El Cayo permitiría determinar como esta especie contribuye a la estructura de la comunidad asociada a la vegetación sumergida y ayudaría a establecer el papel regulador de estos organismos dentro de la comunidad (Licona y Moreno 1996). Los estudio de las relaciones tróficas y de los efectos de la depredación que ejerce L. griseus sobre otras especies de alto valor comercial como camarones y cangrejos, son de especial interés. Estos estudios pueden realizarse mediante el análisis de las proporciones de los componentes alimenticios dominantes presentes en la dieta de los depredadores (DíazGonzález y Soto 1988).
Las relaciones tróficas de especies de la familia Lutjanidae han sido estudiadas en zonas costeras con diversos hábitats (González Sansón y Rodríguez 1985, Harrigan et al. 1989, Hettler 1989, Aguilar et al. 1992b, Rooker 1995, Sierra 1996-1997). En la Laguna de Términos se han realizado importantes estudios de las comunidades que habitan en fondos con vegetación sumergida, algunos incluyen la biología de varias especies (Yáñez-Arancibia et al. 1982, Yáñez-Arancibia y Lara-Domínguez 1983). Rivera (1990) estudió los hábitos alimenticios de Lutjanus synagris (Linnaeus, 1758) en las entradas de la laguna, Zárate (1996) informa que L. griseus es una especie dominante en las zonas de manglares y Sánchez (1993, 1994) estudió las relaciones tróficas de L. apodus (Walbaum 1792) en áreas con vegetación sumergida. Sin embargo, hasta el momento no han sido estudiadas las relaciones tróficas de $L$. griseus en la Laguna de Términos.

El objetivo del presente estudio fue determinar las relaciones tróficas de L. griseus y los cambios en sus hábitos alimenticios a través de su desarrollo ontogénico. Así como definir las relaciones entre esta especie y la vegetación sumergida en el área de estudio, lo que se llevó a cabo a través del análisis de las variaciones del contenido estomacal de $L$. griseus en escalas espacial y temporal. Este estudio se basó en un esquema de muestreo intensivo que permitió obtener una muestra amplia y representativa de esta especie.

\section{MATERIALES Y MÉTODOS}

Área de estudio: la Laguna de Términos se localiza en el sur del Golfo de México, se comunica con la Sonda de Campeche mediante las bocas del Carmen y Puerto Real, en una zona tropical, por lo que no presenta una fuerte variación estacional. Sin embargo, diversos estudios ecológicos realizados en el área (Yáñez-Arancibia y Day 1982, YáñezArancibia et al. 1983) sugieren tres periodos climáticos en relación con las variaciones en 
las precipitaciones, descarga de los ríos, frecuencia de los vientos y temperatura ambiente. Estas épocas climáticas son: época de secas de febrero a mayo, época de lluvias persistentes en la tarde y noche durante junio a octubre y la época de nortes con lluvias ocasionales y fuertes vientos del norte de octubre a enero.

Existe una gran variedad de ambientes estuarinos en la laguna, incluyendo vegetación sumergida en su porción norte, donde se presenta una persistente influencia marina, lo que mantiene valores elevados de salinidad y transparencia. En esta área se localiza El Cayo (Fig. 1) que comprende una extensión aproximada de $52900 \mathrm{~m}^{2}$, la vegetación sumergida está compuesta de pastos marinos, dominados por la fanerógama Thalassia testudinum, apareciendo en menor densidad Halodule wrightii, Syringodium filiforme y macroalgas como Phaephyceae y Rhodophyceae, la vegetación circundante está formada por árboles de mangle, predominando en el área sedimentos arenosos y limosos (Sánchez 1994).

En esta localidad se registran durante todo el año densidades elevadas de macro crustáceos, particularmente camarones peneidos y cangrejos anomuros y braquiuros (Raz-Guzmán et al. 1986, Álvarez et al. 1987, Raz-Guzmán y Sánchez 1992, Sánchez y Raz-Guzmán 1992), aunque también se encuentran presentes muchos peces (Vargas-Maldonado et al. 1981,

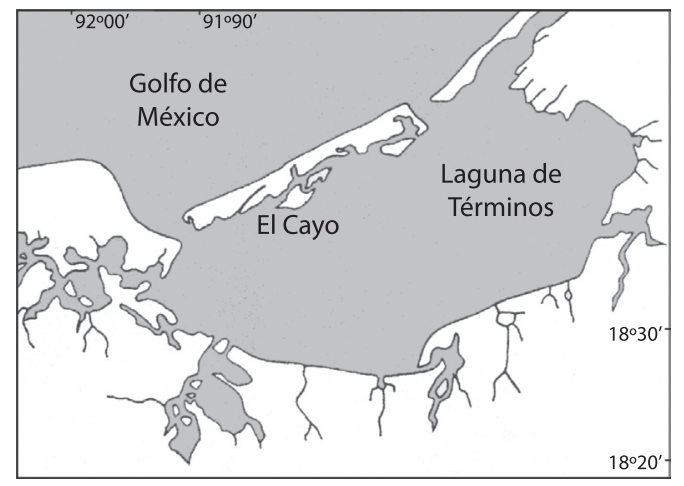

Fig. 1. Laguna de Términos en Campeche, México. Se indica la localidad El Cayo, donde se realizaron las capturas de peces.
Yáñez-Arancibia y Lara-Domínguez 1983) y productores primarios (Day et al. 1982).

Trabajo de campo y análisis de muestras: los peces estudiados en esta investigación se recolectaron mediante un diseño de muestreo que incluyó la variación espacial entre sustratos con vegetación y sin vegetación y la variación temporal en una escala diaria (diurnos y nocturnos) y otra estacional (seca, lluvias y nortes).

Se efectuaron mensualmente cuatro colectas de la siguiente forma: en una zona con fondos de vegetación sumergida y en condiciones de iluminación (VI), en una zona sin vegetación y en condiciones de iluminación (SVI), en una zona de vegetación y en condiciones de oscuridad (VO) y en una zona sin vegetación y en condiciones de oscuridad (SVO). En total resultaron 48 colectas de peces de las cuales $50 \%$ fueron diurnas y $50 \%$ nocturnas, desde septiembre de 1997 hasta agosto de 1998, incluyendo las tres épocas del año, secas, lluvias y nortes.

Las colectas se realizaron mediante una red de arrastre de prueba camaronera de $5 \mathrm{~m}$ de largo, $2.5 \mathrm{~m}$ de abertura de trabajo y luz de malla de $1.25 \mathrm{~cm}$, equipada con tablas de 0.8 por $0.5 \mathrm{~m}$ y operada durante $10 \mathrm{~min}$ por una lancha a una velocidad de $0.5 \mathrm{~ms}^{-1}$, cubriendo un área de arrastre aproximada de $1500 \mathrm{~m}^{2}$.

En cada recolecta se tomaron los valores de salinidad de superficie y fondo con un refractómetro American Optical de lectura directa y temperatura compensada con precisión de $1 \%$. La temperatura superficial y de fondo fue medida con un termómetro de cubeta marca Kahlsico con precisión de $0.1^{\circ} \mathrm{C}$.

Todos los ejemplares de L. griseus recolectados fueron medidos (longitud total, LT en $\mathrm{mm}$ ) y pesados (peso húmedo en $\mathrm{g}$ ), se les extrajo el estómago que se conservó en formol al $10 \%$. Los componentes alimenticios fueron separados por categorías taxonómicas. El análisis de los contenidos estomacales se realizó mediante los métodos numérico, frecuencial y gravimétrico según la metodología de Rosecchi y Novaze (1987). Se calcularon los índices de importancia relativa de Pinkas et al. (1971), cuya expresión es: $\mathrm{RII}=(\mathrm{N}+\mathrm{G}) \mathrm{F}$, donde $\mathrm{N}$ es 
el porcentaje numérico, $\mathrm{G}$ es el porcentaje gravimétrico y $\mathrm{F}$ es el porcentaje frecuencial y el índice de amplitud o diversidad de dietas (Levins 1968, Evans 1983), cuya expresión es: $\mathrm{B}=\left(\Sigma \mathrm{p}_{\mathrm{i}}^{2}\right)^{-1}$, donde $\mathrm{p}_{\mathrm{i}}$ es la proporción numérica de una categoría trófica con relación al total y el valor de $B$ varía desde uno hasta $n$, siendo $\mathrm{n}$ el número de categorías tróficas encontradas en los estómagos. Este índice se calculó para seis clases de talla de L. griseus durante los tres periodos climáticos. Como los valores de $\mathrm{n}$ no resultaron iguales para todas las clases de talla los valores de B se normalizaron según la expresión Bs=B-1/n-1 (Evans 1983).

La abundancia de $L$. griseus se estimó en términos de densidad (número de individuos $\mathrm{m}^{-2}$ ) y biomasa ( $\mathrm{g}$ de peso húmedo $\mathrm{m}^{-2}$ ). Con estos valores calculados en cada mes para las cuatro condiciones de muestreo (VI, SVI, VO y SVO) se realizó una prueba de correlación múltiple y análisis de varianza para comprobar si la salinidad y la temperatura promedios de la zona de estudio influyó en la abundancia de la especie.

\section{RESULTADOS}

Influencia de la salinidad y la temperatura en la distribución mensual de L. griseus: Las variaciones mensuales en el número de individuos de cada una de las seis clases de talla mostraron que durante mayo, junio y julio de 1998 hubo un incremento en el número de individuos mayores de $131 \mathrm{~mm}$ de LT (Fig. 2). En contraste, los individuos menores de $100 \mathrm{~mm}$ de LT fueron más frecuentes de septiembre a noviembre de 1997 que durante el resto de los meses. Los cambios en la distribución de las clases de talla mostraron que los peces menores de $70 \mathrm{~mm}$ de LT encontrados en noviembre incrementaron sus tallas hasta 71-100 y 101-130 mm de LT durante los meses subsiguientes.

La temperatura media del agua varió desde $26{ }^{\circ} \mathrm{C}$ en diciembre de 1997 y enero de 1998 hasta $32{ }^{\circ} \mathrm{C}$ en agosto de 1998 , mientras que la salinidad incrementó de $22 \%$ en noviembre de 1997 a $40 \%$ en junio de 1998 (Fig. 3). La correlación entre la temperatura del agua y la biomasa de los peces fue significativa en las condiciones VI, SVI y VO $\left(\mathrm{r}^{2}=0.6765\right.$, $\mathrm{p}=0.0010 ; \mathrm{r}^{2}=0.4155, \mathrm{p}=0.0236 ; \mathrm{r}^{2}=0.4738$, $\mathrm{p}=0.0133$, Cuadro 1). La correlación entre la salinidad y la biomasa también resultó significativa en las condiciones VI, SVI y VO $\left(\mathrm{r}^{2}=0.6203, \mathrm{p}=0.0023 ; \mathrm{r}^{2}=0.3809, \mathrm{p}=0.0325\right.$; $\mathrm{r}^{2}=0.7787, \mathrm{p}=0.0001$ ). Entre la temperatura y la densidad de los peces resultó significativa solamente en la condición SVI $\left(\mathrm{r}^{2}=0.4378\right.$, $\mathrm{p}=0.0190$ ), mientas que la correlación entre la salinidad y la densidad fue significativa en las condiciones SVI y VO $\left(\mathrm{r}^{2}=0.3778, \mathrm{p}=0.0334\right.$; $\mathrm{r}^{2}=0.8265, \mathrm{p}=0.0004$; Cuadro 1).

La comparación mensual de las variables poblacionales mostró que la densidad expresada en número de individuos $\mathrm{m}^{-2}$ y la biomasa expresada como $\mathrm{g}$ de peso húmedo $\mathrm{m}^{-2}$ presentaron tendencias similares (Fig. 3). Desde mayo a julio de 1998, tanto el número como la talla de los peces recolectados fue mayor que en el resto de los meses analizados (Fig. 2), por lo que el peso medio de la población fue más alto. Las clases de talla mayores no estuvieron representadas durante septiembre, octubre y noviembre de 1997, cuando fueron abundantes los individuos de tallas pequeñas (Fig. 2). Además, el número de peces disminuyó de agosto a diciembre, resultando un menor peso medio de la población hacia finales de año. La comparación entre los periodos de iluminación y oscuridad mostró que el incremento de la densidad en la condición VI durante abril a agosto no estuvo acompañada por cambios marcados en la biomasa, sugiriendo que el incremento en la densidad de peces en la condición VI se debió a la abundancia de organismos pequeños que contribuyen en menor medida a la biomasa de la población.

Distribución de L. griseus y preferencia de hábitat: del total de individuos de L. griseus recolectados en este estudio, $83 \%$ fueron capturados en áreas con vegetación durante la noche y $16 \%$ en áreas con vegetación durante el día. El mayor porcentaje de peces con 
CUADRO 1

Análisis de varianza de la correlación entre la temperatura y la salinidad con la densidad y biomasa de Lutjanus griseus en cuatro condiciones de muestreo

\begin{tabular}{|c|c|c|c|c|c|}
\hline Correlación & Condición* & $r^{2}$ & $d f$ & $F$ & $P$ \\
\hline Temperatura-biomasa & VI & 0.6765 & 1,10 & 20.915 & 0.0010 \\
\hline Temperatura-biomasa & SVI & 0.4155 & 1,10 & 7.109 & 0.0236 \\
\hline Temperatura-biomasa & $\mathrm{VO}$ & 0.4738 & 1,10 & 9.006 & 0.0133 \\
\hline Temperatura-biomasa & SVO & 0.1468 & 1,10 & 1.721 & 0.2180 \\
\hline Salinidad-biomasa & VI & 0.6203 & 1,10 & 16.338 & 0.0023 \\
\hline Salinidad-biomasa & SVI & 0.3809 & 1,10 & 6.152 & 0.0325 \\
\hline Salinidad-biomasa & $\mathrm{VO}$ & 0.7787 & 1,10 & 35.197 & 0.0001 \\
\hline Salinidad-biomasa & SVO & 0.2140 & 1,10 & 2.722 & 0.2299 \\
\hline Temperatura-densidad & VI & 0.0019 & 1,10 & 0.019 & 0.8918 \\
\hline Temperatura-densidad & SVI & 0.4378 & 1,10 & 7.788 & 0.0190 \\
\hline Temperatura-densidad & VO & 0.2804 & 1,10 & 3.896 & 0.0766 \\
\hline Temperatura-densidad & SVO & 0.0610 & 1,10 & 0.650 & 0.4386 \\
\hline Salinidad-densidad & VI & 0.0178 & 1,10 & 0.182 & 0.6786 \\
\hline Salinidad-densidad & SVI & 0.3778 & 1,10 & 6.072 & 0.0334 \\
\hline Salinidad-densidad & $\mathrm{VO}$ & 0.8265 & 1,10 & 47.662 & 0.0004 \\
\hline Salinidad-densidad & SVO & 0.2108 & 1,10 & 2.671 & 0.1331 \\
\hline
\end{tabular}

\footnotetext{
* Fondos de vegetación sumergida y en condiciones de iluminación (VI), zonas sin vegetación y en condiciones de iluminación (SVI), zonas de vegetación y en condiciones de oscuridad (VO) y en zonas sin vegetación y en condiciones de oscuridad (SVO).
}

alimento en sus estómagos (76 \%) se encontró en las muestras recolectadas en la condición VO. En contraste, en las zonas sin vegetación se capturó solamente 0.9 y $0.3 \%$ de los peces durante la noche y el día respectivamente (Cuadro 2).

Composición de la dieta: del total de peces recolectados, $68 \%$ (672 individuos) tenían alimento en sus estómagos. En la Cuadro 3 se presenta la composición de la dieta de $L$. griseus expresada en porcentaje numérico, gravimétrico y frecuencial, así como el índice de importancia relativa (IIR). Los organismos evasivos bentónicos como Farfantepenaeus duorarum, carideos, Hyppolyte zostericola, y
Palemonetes octaviae, junto con restos de crustáceos no identificados presentaron el mayor valor del IIR (14 282), seguido por los organismos nadadores tales como Eucinostomus gula (4 277). Los organismos reptantes del bentos como el cangrejo Libinia dubia presentaron los menores valores del índice (1 143).

Variaciones de la dieta de acuerdo al desarrollo ontogénico: los individuos menores de $100 \mathrm{~mm}$ de LT se alimentaron principalmente de organismos evasivos bentónicos, de los cuales los camarones fueron los más abundantes (Fig. 4). Los peces de las clases de talla intermedias (101-160 mm de LT) se alimentaron, además de organismos nadadores 

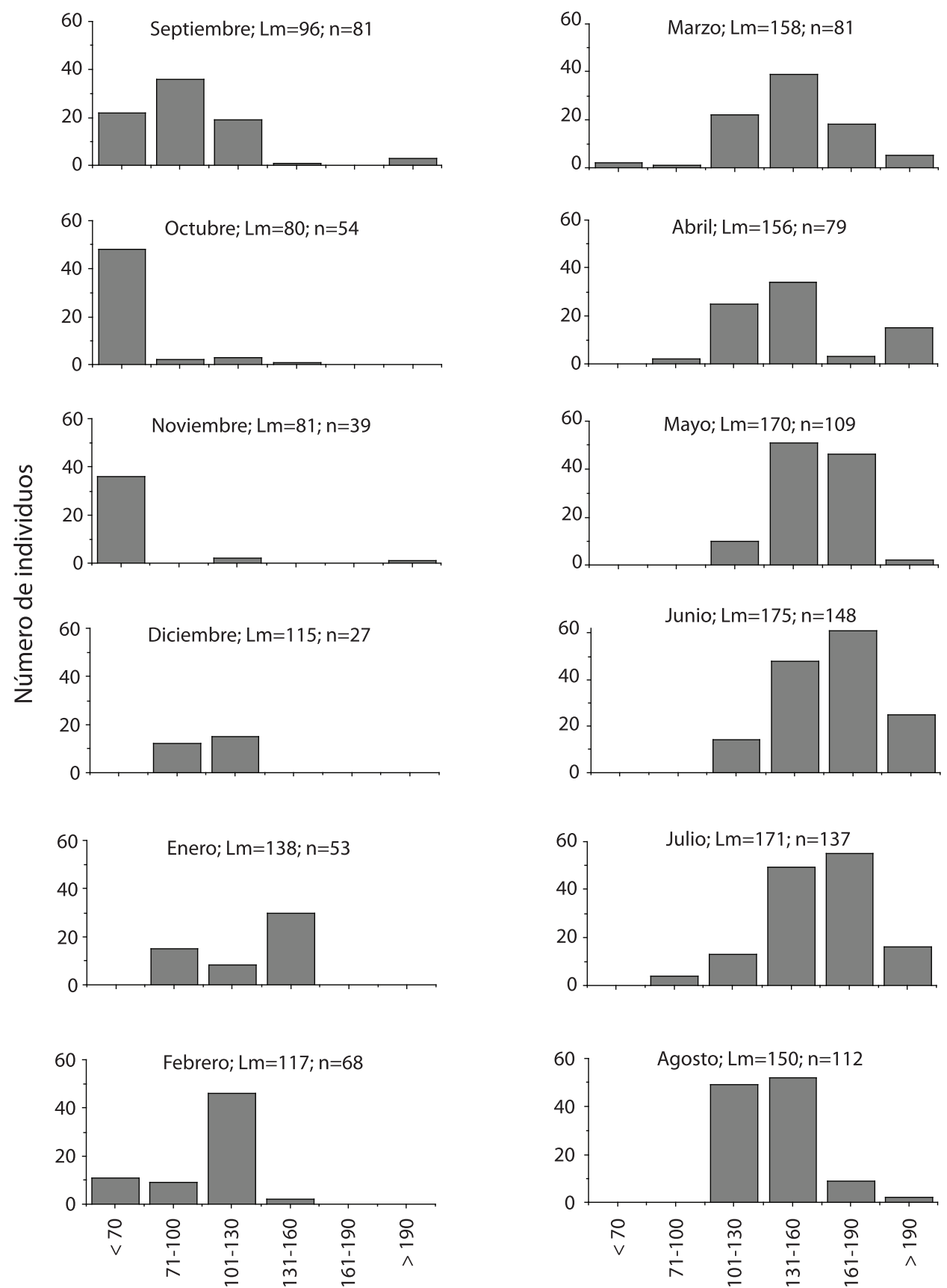

\section{Largo total (mm)}

Fig. 2. Variaciones mensuales en número de seis clases de talla de Lutjanus griseus, Lm= largo total medio (mm), $n=$ número de individuos. 


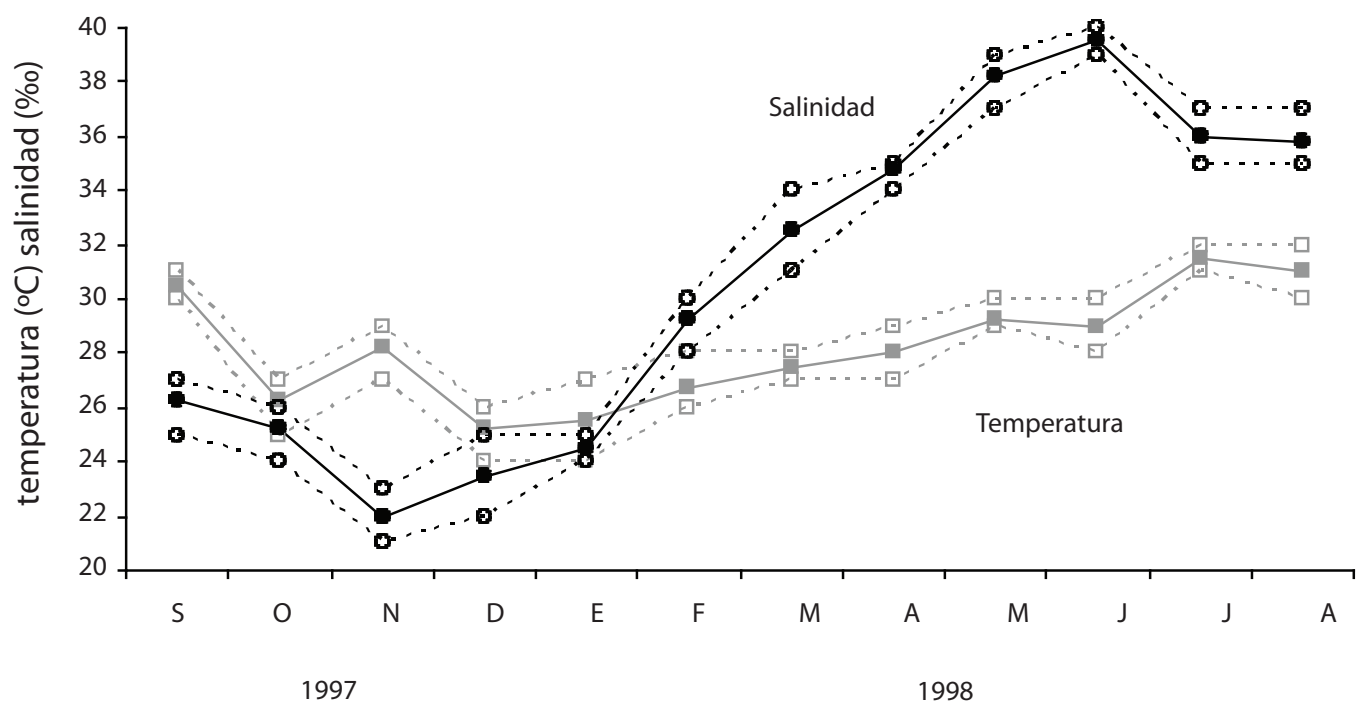

Meses
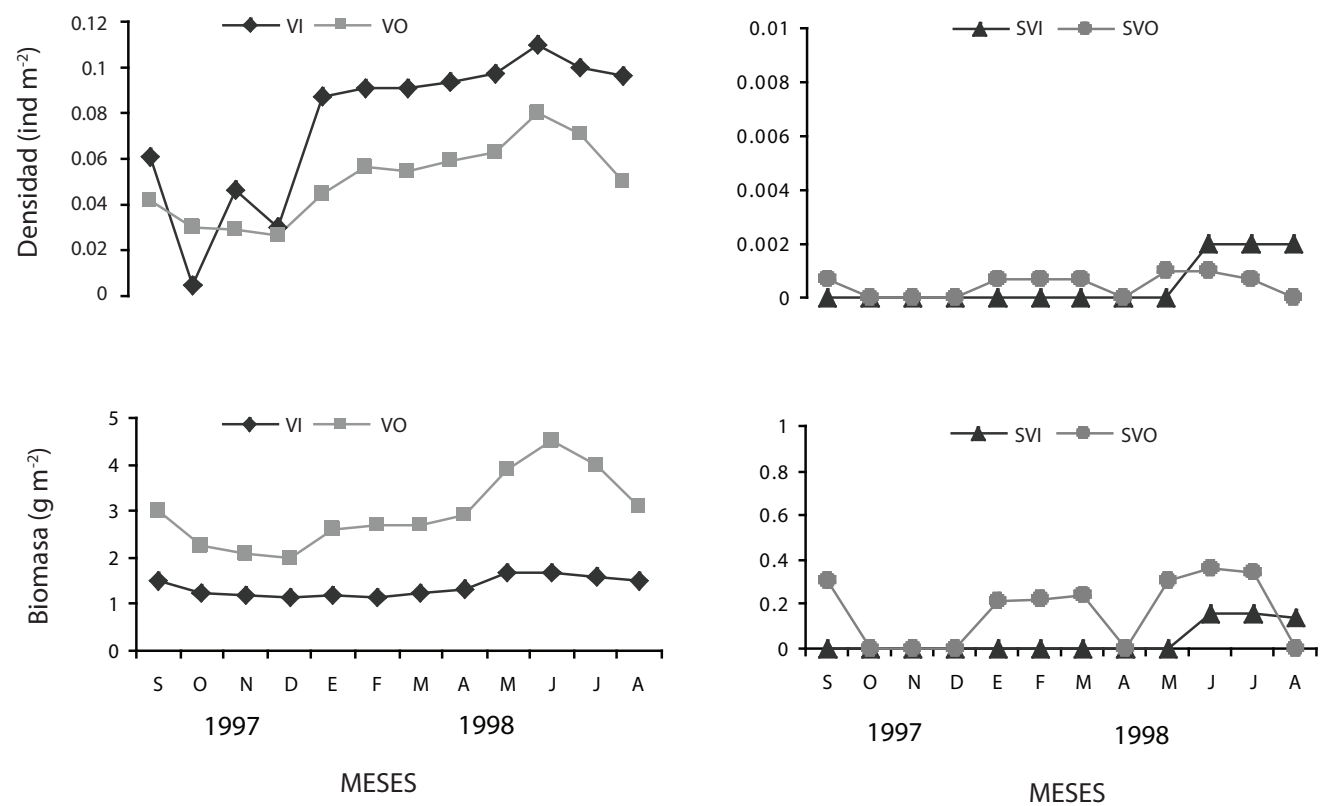

Fig. 3. Variaciones mensuales de la salinidad $\left(\%\right.$ oo y la temperatura $\left({ }^{\circ} \mathrm{C}\right)$ durante un año de muestreo, valores máximos y mínimos representados por símbolos claros, valores medios representados por símbolos oscuros. Abundancia de Lutjanus griseus expresada en número de individuos $\mathrm{m}^{-2} \mathrm{y} \mathrm{g} \mathrm{m}^{-2}$ en cuatro condiciones de muestreo: zonas con vegetación durante el día (VI), zonas con vegetación durante la noche (VO), zonas sin vegetación durante el día (SVI) y zonas sin vegetación durante la noche (SVO). 
CUADRO 2

Variaciones temporales y espaciales en la abundancia de Lutjanus griseus en El Cayo

Zonas con vegetación

Meses

Septiembre 1997
Octubre
Noviembre
Diciembre
Enero 1998
Febrero
Marzo
Abril
Mayo
Junio
Julio
Agosto

Grupos tróficos

Organismos evasivos del bentos

(peneidos, carideos y restos de crustáceos no identificados)

Organismos nadadores

(peces y restos de peces no identificados)

Organismos reptantes del bentos (Libinia dubia)

y de organismos reptantes del bentos. En los individuos mayores de $160 \mathrm{~mm}$ de LT, los peces fueron el principal componente alimenticio encontrado. En general se observó un incremento en el consumo de peces a medida que aumentó la talla de L. griseus (Fig. 4).

La comparación del contenido estomacal de peces capturados durante los periodos de iluminación y oscuridad mostró un mayor
Zonas sin vegetación

Día

Noche

$\begin{array}{cccc}\begin{array}{c}\text { Día } \\ \text { No. de } \\ \text { individuos }\end{array} & (\%) & \begin{array}{c}\text { No. de } \\ \text { individuos }\end{array} & (\%) \\ - & - & 1 & 1 \\ 1 & 2.5 & 1 & 2.5 \\ - & - & - & - \\ - & - & - & - \\ - & - & 2 & 3.7 \\ 1 & 1.4 & - & - \\ - & - & - & - \\ - & - & 2 & 2 \\ - & - & 1 & 1 \\ 1 & 1 & - & - \\ - & - & - & - \\ - & - & 2 & 2\end{array}$

CUADRO 3

Contenido estomacal de Lutjanus griseus, porcentages numérico, en peso y frecuencial y el Índice de Importancia Relativa (IIR) según Pinkas (1971)

$\begin{array}{cccc}\begin{array}{c}\% \text { en } \\ \text { número } \\ \mathrm{N}\end{array} & \begin{array}{c}\text { \% en } \\ \text { peso } \\ \mathrm{W}\end{array} & \begin{array}{c}\text { \% en } \\ \text { frecuencia } \\ \mathrm{F}\end{array} & \begin{array}{c}\mathrm{IIR} \\ \mathrm{F}(\mathrm{N}+\mathrm{W})\end{array} \\ 65.0 & 42.2 & 133.2 & 14282 \\ 20.3 & 35.8 & 76.2 & 4277 \\ 14.7 & 21.9 & 31.3 & 1143\end{array}$

consumo de organismos nadadores durante las horas del día que durante la noche. Por el contrario, los organismos evasivos y reptantes del bentos fueron consumidos en mayor proporción durante las horas de la noche.

Los valores del índice de amplitud o diversidad de dietas (Bs) para las seis clases de talla de L. griseus variaron con la talla de los peces y durante las tres épocas climáticas 

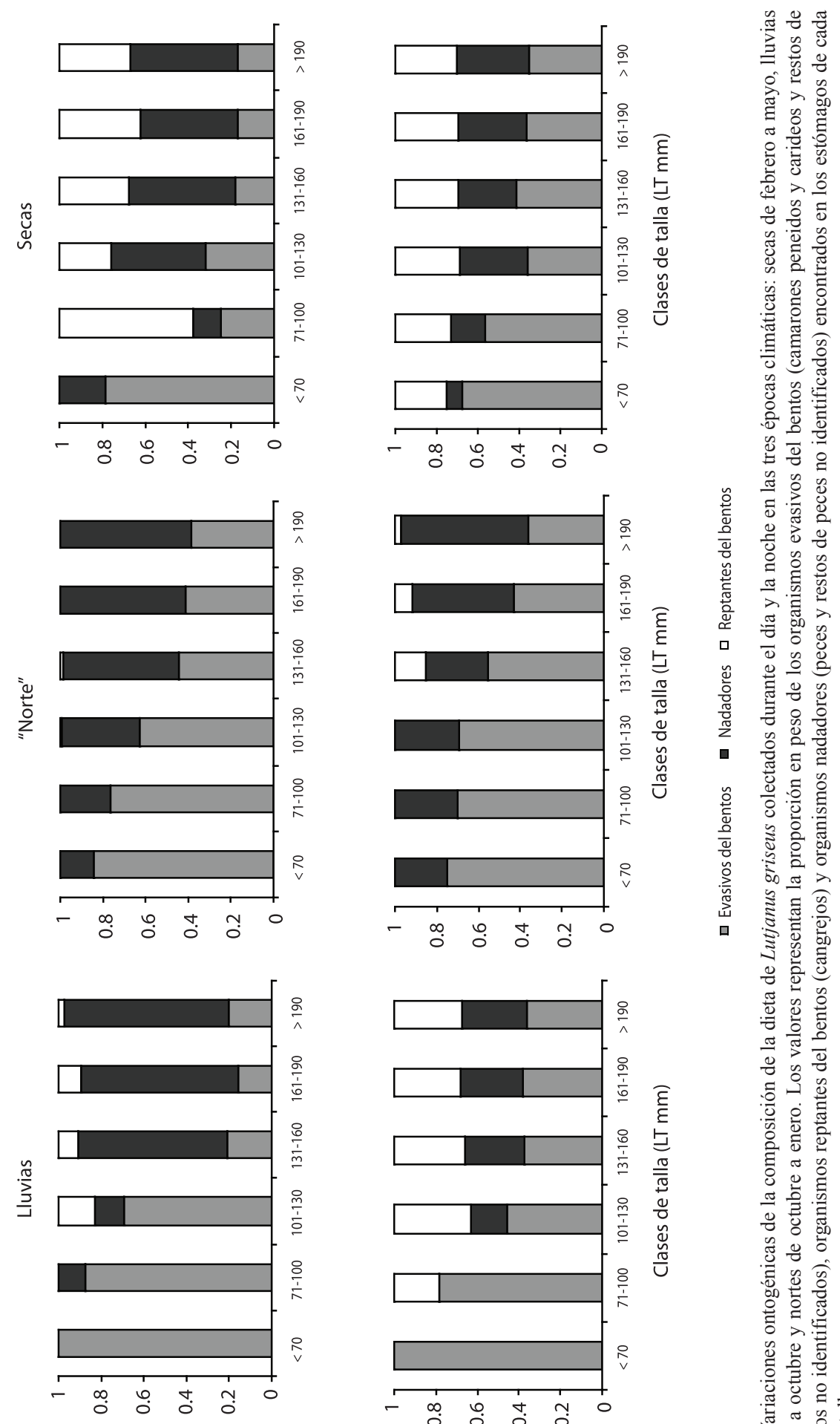

(6) osəd uə uọ!sıodold

$\forall I O$

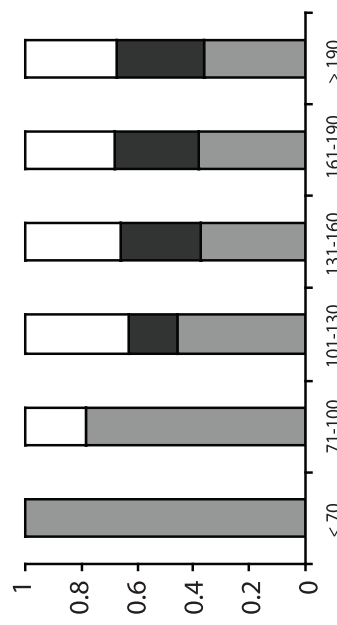

(6) osəd uə uọ!̣ıodoxd ヨHOON

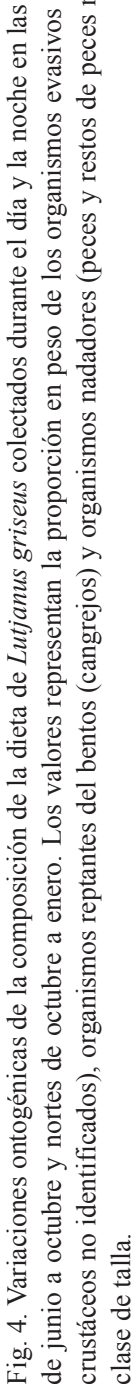

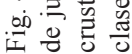




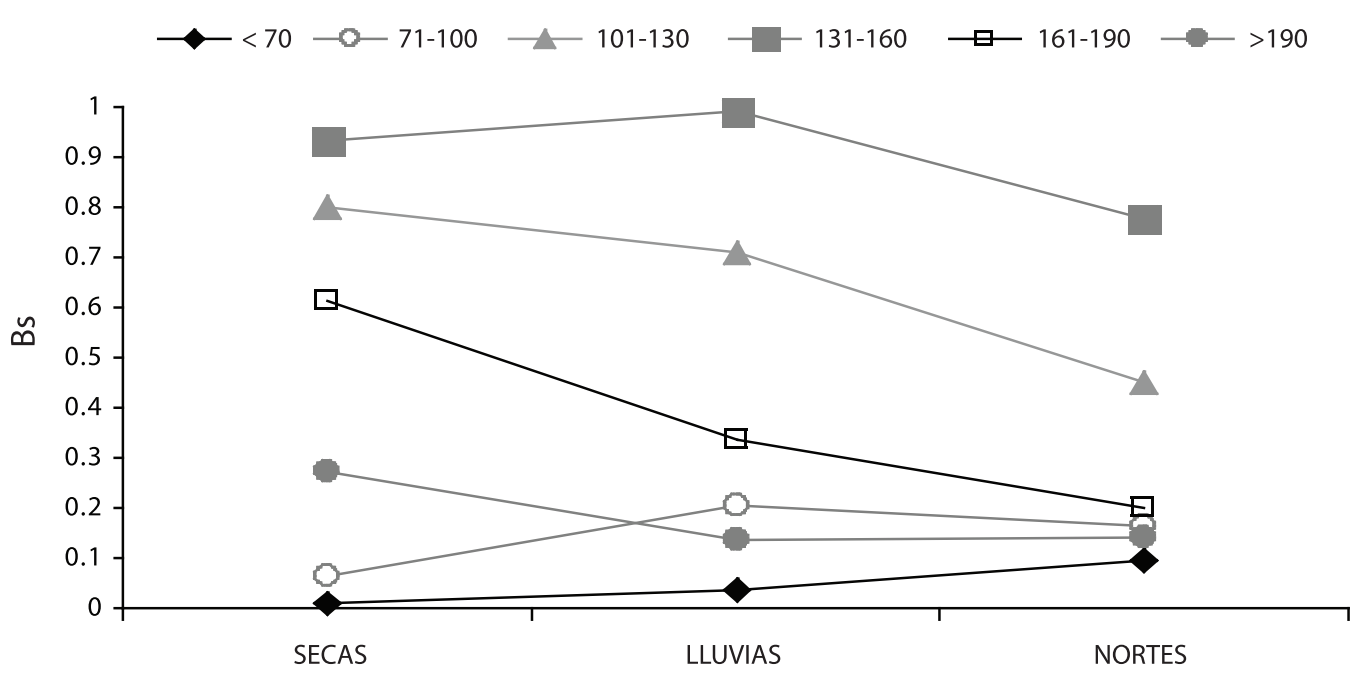

Estaciones

Fig. 5. Valores del índice de diversidad de dieta normalizado (Bs) para seis clases de talla de Lutjanus griseus. Época de secas de febrero a mayo, época de lluvias de junio a octubre, época de nortes de octubre a enero.

(Fig. 5). En los peces más pequeños (menores de $100 \mathrm{~mm}$ de LT) y en los más grandes (mayores de $190 \mathrm{~mm}$ de LT) se encontraron valores más bajos de Bs que en los individuos de tallas intermedias durante las tres épocas del año. Mientras que los valores de Bs calculados para los individuos más pequeños (menores de $100 \mathrm{~mm}$ de LT) y para los más grandes (mayores de $190 \mathrm{~mm}$ de LT) permanecieron relativamente constantes a través del año, los valores de Bs para los peces de tallas intermedias (101-190 mm LT) decrecieron de la estación de secas a la de nortes (Fig. 5).

\section{DISCUSIÓN}

Según Hettler (1989), L. griseus puede utilizar las zonas de canales de mangle como refugio durante el día y los pastos para forrajear en la noche. En correspondencia con esto el $83 \%$ de los peces recolectados se encontraron de noche, casi todos con algún alimento en su estómago. Esta especie ha sido señalada como dominante donde interaccionan fanerógamas marinas y manglar (Yáñez-Arancibia y Lara-Domínguez 1983, Yáñez-Arancibia et al. 1985a).
Estudios previos identifican a Cathorups melanopus (Günther 1864) (Lara-Domínguez et al. 1981), Cichlasoma urophthalmus (Günther 1862) (Caso Chávez et al. 1986) y Anchoa mitchilli (Hildebrand 1943) (Ayala-Pérez et al. 1997) como las especies de peces dominantes en diferentes áreas de la Laguna de Términos y sistemas adyacentes. Sin embargo Zárate (1996) señala a L. griseus como la especie más abundante y frecuente en los canales de mangle que rodean El Cayo. Este autor informa que $L$. griseus es residente permanente en la zona y que utiliza las áreas de mangle para alimentarse y reproducirse.

Mientras que la talla media de L. griseus en El Cayo aumentó desde octubre de 1997 a junio y julio de 1998, su abundancia decreció de agosto a diciembre de 1997, incrementándose nuevamente en enero del siguiente año. Este patrón de variación de las tallas pudiera ser el resultado de la migración hacia los canales de mangle de los individuos mayores durante el periodo de lluvias, mientras que los peces pequeños se reclutan a El Cayo en septiembre, alcanzando su desarrollo máximo al utilizar las condiciones favorables que ofrece esta área. 
Günther (1961), Pauly (1975) y Castro Aguirre (1982) señalan la gran influencia de las variaciones ambientales en la estructura y dinámica de las comunidades estuarinas. Según Raz-Guzmán (1995), la distribución de las comunidades en los sistemas estuarinos tropicales está regulada por factores que controlan su estructura como la temperatura, la salinidad, el sustrato y los procesos biológicos, así como por las interacciones entre ellos. La temperatura y la salinidad son los principales factores ecológicos que actuando de manera separada o en conjunto, modifican la estructura, funcionamiento y distribución de los organismos marinos y estuarinos (Kinne 1971).

El reclutamiento de postlarvas de peneidos a la laguna durante todo el año (ArenasMendieta y Yáñez-Martínez 1981) y su asentamiento en las zonas de vegetación de la región nor-central de la laguna (Sánchez y Soto 1982), además de la abundancia permanente de otros decápodos como cangrejos anomuros y braquiuros (Sánchez 1994) y juveniles de peces (Vargas-Maldonado et al. 1981, 1987, YáñezArancibia et al. 1985b), hacen a El Cayo un área con alta disponibilidad de alimento para los peces depredadores. Raz-Guzmán (1995) encontró que Eucinostomus gula y juveniles de peneidos son componentes numéricamente importantes de la fauna de esta comunidad.

La correlación significativa entre la abundancia de L. griseus y las variaciones de la salinidad y la temperatura encontradas en este trabajo indican la influencia de estos parámetros ambientales en la abundancia de esta especie en El Cayo y constituye una evidencia de cómo influyen estos factores en las poblaciones de peces de las regiones tropicales. Sin embargo, las altas densidades de macrocrustáceos y peces presentes durante todo el año constituyen una importante fuente de alimento para $L$. griseus, mientras la alta complejidad física de la vegetación sumergida provee a los jóvenes de la especie de refugio efectivo ante sus depredadores.

Al estudiar los hábitos alimenticios de Lutjanus synagris en las bocas que conectan Laguna de Términos con el mar, Rivera
(1990) encontró que estos peces se alimentan con mayor intensidad en horas de la noche. Un comportamiento similar describió Sánchez (1994) para Lutjanus apodus. Los resultados de este trabajo mostraron una marcada preferencia de L. griseus por áreas con vegetación sumergida así como un incremento de su abundancia durante la noche. Varios autores (Greening y Livingston 1982, Fernández 1985, Sánchez 1993) se han referido a la mayor actividad de muchos macrocrustáceos en horas de la noche. Esto se refleja en la mayor incidencia de restos de crustáceos en los estómagos de los peces que fueron recolectados durante la noche en este estudio, mientras que el mayor consumo de peces se observó durante el día. Estos resultados nos brindan evidencia de la depredación nocturna de L. griseus sobre diversas especies de crustáceos.

La vegetación acuática como parte del sustrato es importante en la determinación de la abundancia, la riqueza de especies y de la diversidad de las comunidades estuarinas (Heck y Orth 1980). Raz-Guzmán (1995) demostró que las áreas con vegetación sumergida en la Laguna de Términos soportan comunidades con mayor densidad y complejidad que las áreas que no presentan vegetación. En estudios anteriores se ha mostrado que la vegetación acuática incrementa la heterogeneidad, la complejidad cualitativa, así como la producción primaria y secundaria (Heck y Wetshore 1977). En el presente trabajo se confirman estos resultados ya que el $83 \%$ de los peces fueron capturados en áreas con vegetación y durante la noche, de los cuales el $71 \%$ presentó alimento en sus estómagos, lo que indica que L. griseus utiliza preferentemente para alimentarse las zonas con vegetación en horas de la noche, cuando la abundancia de diferentes presas es alta.

Los lutjanidos se alimentan en general de crustáceos y peces (González-Sansón y Rodríguez 1985, Yáñez-Arancibia y Sánchez Gil 1986, Harrigan et al. 1989, Hettler 1989, Rivera 1990, Aguilar et al. 1992b, Sánchez 1994, Guevara et al. 1994, Rooker 1995, Sierra 1996-1997). En este trabajo los principales 
grupos tróficos encontrados en los estómagos de los individuos pequeños fueron organismos evasivos del bentos (camarones peneidos y carideos así como restos de crustáceos no identificados), sin embargo, en los individuos mayores Eucinostomus gula y los restos de peces no identificados fueron las entidades dominantes. Todos estos componentes alimenticios son abundantes en los hábitats con vegetación sumergida en El Cayo durante todo el año.

Aunque los camarones peneidos constituyeron el componente alimenticio dominante de $L$. griseus, los peces formaron parte importante de la dieta en los individuos mayores de $150 \mathrm{~mm}$ de LT (Harrigan et al. 1989, Hettler 1989). Al incrementar su talla, los peces fueron el taxon dominante en las presas de Lutjanus apodus (Rooker 1995). En este estudio también se encontró un cambio de la dieta en relación al desarrollo ontogénico. Los individuos mayores consumieron más peces (organismos nadadores) que los individuos de tallas intermedias y pequeñas, las tallas intermedias de L. griseus se alimentaron de los tres grupos tróficos (organismos evasivos y organismos reptantes del bentos y organismos nadadores), mientras que los individuos de las tallas más pequeñas se alimentaron de organismos evasivos del bentos.

Debido a que la talla media de L. griseus incrementó durante el año, las variaciones mensuales en la composición de la dieta también reflejan las variaciones de la dieta al aumentar la talla de los peces. La mayor abundancia de individuos pequeños (menores de $70 \mathrm{~mm}$ de LT) se observó en septiembre, octubre y noviembre de 1997, durante los cuales los organismos evasivos del bentos fueron encontrados más frecuentemente en sus estómagos. Estos individuos comenzaron a crecer en diciembre de 1997, siendo los peces de las tallas intermedias (71-100, 101-130 y 131-160 mm de LT) más abundantes de enero a abril de 1998. Durante este periodo L. griseus se alimentó de diferentes grupos tróficos que incluyeron crustáceos y peces. En mayo, junio y julio, los peces alcanzaron las clases de tallas mayores (161-190 y más de 190 mm de LT) y se alimentaron de organismos nadadores (peces y restos de peces no identificados) en mayor proporción que durante los otros meses.

Al analizar el índice de amplitud de dieta normalizado (Bs) se encontró que en los peces de tallas intermedias (101-130, 131-160 y 160$190 \mathrm{~mm}$ de LT) el índice alcanzó valores más altos que en los individuos pequeños (menos de $100 \mathrm{~mm}$ de LT) y mayores (más de 190 $\mathrm{mm}$ de LT), resultados que concuerdan con las variaciones observadas en la composición de la dieta durante el desarrollo ontogénico, ya que los individuos de tallas intermedias se alimentaron de las tres categorías tróficas (organismos evasivos y reptantes del bentos y organismos nadadores), mientras que los individuos de tallas más pequeñas y mayores se alimentaron solamente de organismos evasivos del bentos y organismos nadadores respectivamente. Los menores valores de Bs correspondieron a los individuos menores de $70 \mathrm{~mm}$ de LT.

En los peces de tallas intermedias (101-130, $131-160$ y $161-190 \mathrm{~mm}$ de LT) los valores de Bs fueron menores durante la estación de nortes que durante las estaciones de lluvia y secas, además, durante diciembre, enero y febrero no se encontraron cangrejos en los estómagos de los individuos de estas tallas. Esto puede explicarse por una disminución en la abundancia de crustáceos, particularmente del cangrejo Libinia dubia durante la estación de nortes.

A pesar de las variaciones en la diversidad de dieta, no se encontró una variación estacional marcada en la dieta de L. griseus, sólo se observaron pequeños cambios en la proporción en número de los componentes tróficos. El hecho de que se hayan encontrado componentes tróficos similares a lo largo de todo un año de estudio es consecuencia de la estabilidad del área, en la que la constante influencia marina y la alta abundancia y diversidad de las comunidades asociadas a la vegetación sumergida provee de una base alimenticia para muchas especies de peces durante alguna parte de su ciclo de vida. 


\section{AGRADECIMIENTOS}

Los autores agradecen a la Estación de Investigaciones Marinas "El Carmen", UNAM por el apoyo en el trabajo de campo y de laboratorio, en especial a Francisco Vera Herrera y Andrés Reda Deara. Emma Guevara recibió una beca de la Secretaría de Relaciones Exteriores de México. Este trabajo fue parcialmente financiado por la Dirección General de Asuntos del Personal Académico de la UNAM (proyecto 214596) y CONACYT (proyecto 3293P-9607).

\section{RESUMEN}

Se presentan los resultados de un estudio de los hábitos alimenticios de Lutjanus griseus asociado a la vegetación sumergida en El Cayo, Laguna de Términos, Campeche, México. La investigación se realizó siguiendo un diseño de muestreo intensivo tanto en escala espacial como temporal. Se recolectaron un total de 994 individuos, de los cuales 672 tenía alimento en sus estómagos. Se analizaron los porcentajes en número, peso y frecuencia de los grupos tróficos, así como el índice de importancia relativa. Los componentes alimenticios dominantes fueron Farfantepenaeus duorarum, Palaemonetes octavie, Eucinostomus gula y Libinia dubia. La salinidad y la temperatura se relacionaron con el número de individuos colectados así como con un incremento en el consumo de peces por los individuos de mayor talla. Se calculó el índice de diversidad de dieta para seis clases de talla de los peces, examinándose su variación a través del año. Los peces de menor y mayor talla mostraron los valores más bajos de este índice, mientras que los organismos de las tallas intermedias presentaron los valores más altos. Los resultados mostraron que L. griseus tiene preferencia por hábitats con vegetación sumergida. El porcentaje de peces con alimento en sus estómagos fue mayor durante la noche que durante el día, evidenciando los hábitos nocturnos de esta especie.

Palabras claves: espectro trofico, peces de estuario, pastos marinos, Lutjanus griseus, Laguna de Términos, México.

\section{REFERENCIAS}

Aguilar, C., G. González Sansón, E. Guevara \& A. Bosch. 1992a. Estructura de las comunidades de peces en la bahía de Cienfuegos y la laguna de Guanaroca, Cuba. Rev. Invest. Mar. 13: 222-232.
Aguilar, C., G. González Sansón \& T. Veledo. 1992b. Alimentación natural de jóvenes de la rabirrubia (Ocyurus chrysurus Bloch) en una zona de la plataforma suroccidental de Cuba. Rev. Invest. Mar. 13: 243-253.

Aguirre-León, A. \& S. Díaz-Ruiz. 2000. Estructura poblacional, madurez gonádica y alimentación de Eugerres plumieri (Gerreidae) en el sistema fluvio-deltaico Pom-Atasta, México. Ciencias Marinas 26: 253-273.

Álvarez, N.F., A. Gracia \& L.A. Soto. 1987. Crecimiento y mortalidad de las fases estuarinas del camarón rosado Penaeus (Farfantapenaeus) duorarum Burkenroad, 1939 en la Laguna de Términos, Campeche, México. An. Inst. Cienc. Mar y Limnol. UNAM 14: 207-220.

Arenas-Mendieta, R. \& A. Yánez-Martínez. 1981. Patrón anual de inmigración de postlarvas de camarón (Crustacea: Decápoda: Penaeidae) en la Boca de Puerto Real, Laguna de Términos, Campeche. Tesis Profesional-Mancomunada Biólogo, Facultad de Ciencias, UNAM, México D.F., México.

Ayala-Pérez, L.A., J.L. Rojas-Galavíz \& O.A. AvilésAlatriste. 1996-1997. Crecimiento, reproducción y dinámica poblacional de Anchoa mitchilli (Pisces: Engraulidae) en el sistema Palizada-Del Este, Campeche, México. Rev. Biol. Trop. 44-45: $507-$ 518 .

Botello, A.V., G. Ponce, A. Toledano, G. Díaz \& S. Villanueva. 1992. Ecología, recursos costeros y contaminación en el Golfo de México. Ciencia y Desarrollo 17: 28- 48 .

Caso-Chávez, M., A. Yáñez-Arancibia \& A.L. LaraDomínguez. 1986. Biología, ecología y dinámica de poblaciones de Cichlasoma urophthalmus (Günther) en habitat de Thalassia testudinum y Rhizophora mangle Laguna de Términos, Campeche (Pisces: Cichlidae). Biótica 11: 79-111.

Castro Aguirre, J.L. 1982. Los peces de las lagunas Oriental y Occidental, Oaxaca, México y sus relaciones con la temperatura y salinidad. II. Análisis multifactorial. Anales de la Escuela Nacional de Ciencias Biológicas, México 26: 85-100.

Díaz-González, G. \& L.A. Soto. 1988. Hábitos alimenticios de peces depredadores del sistema lagunar Huizache-Caimanero, Sinaloa, México. An. Inst. Cienc. Mar y Limnol., UNAM 15: 97-124.

Day, J.W. Jr, R. Day, M.T. Barreiro, F. Ley-Lou \& C.J. Madden. 1982. Primary production in the Laguna de 
Terminos, a tropical estuary in the Southern Gulf of Mexico. In P. Lasserre \& H. Postma (eds.). Coastal Lagoons. Oceanológica Acta Vol. Spec. 5: 269-276.

Evans, S. 1983. Production, predation and food niche segregation in a marine shallow soft-botton community. Mar. Ecol. Prog. Ser. 76: 249-260.

Fernández, M.L. 1985. Estudio comparativo de la epifauna asociada a praderas de Thalassia testudinum entre los ambientes marino y lagunar en la costa de Campeche, México. Tesis Profesional Biólogo, Facultad de Ciencias, UNAM, México D.F., México.

González-Sansón, G. \& J. Rodríguez. 1985. Alimentación natural de algunas especies de peces depredadores en las lagunas costeras de Tunas de Zaza, Cuba. Rev. Invest. Mar. 6: 91-99.

Greening, H.S. \& R.J. Livingston. 1982. Diel variation in the structure of seagrass-associated epibenthic macroinvertebrate communities. Mar. Ecol. Prog. Ser. 7: 147-156.

Guevara, E., A. Bosch, R. Suárez \& R. Lalana. 1994. Alimentación natural de tres especies de pargos (Pisces: Lutjanidae) en el Archipiélago de los Canarreos, Cuba. Rev. Invest. Mar. 15: 63-72.

Günther, G. 1961. Some relations of estuarine organism to salinity. Limnol. Oceanograph. 6: 183-190.

Harrigan, P., J.C. Zieman \& S.A. Macko. 1989. The base of nutritional support for the gray snapper (Lutjanus griseus): An evaluation based on a combined stomach content and stable isotope analysis. Bull. Mar. Sci. 44: 65-77.

Heck, K.L. Jr. \& G.S. Wetstone. 1977. Habitat complexity and invertebrate species richness and abundance in tropical seagrass meadows. J. Biogeogr. 4: 135-142.

Heck, K.L. \& R.J. Orth. 1980. Seagrass habitats: the role of habitat complexity, competition and predation in structuring associated fish and motile macroinvertebrate assemblage. In V. Kennedy (ed.). Estuarine Perspectives. Academic, Nueva York, EEUU.

Hettler, W.F. 1989. Food habits of juvenile of spotted seatrout and gray snapper in western Florida Bay. Bull. Mar. Sci. 44: 155-162.

Jensen, K.T. \& J.N. Jensen. 1985. The importance of some epibenthic predators on the density of juveniles benthic macrofauna in the Danish Wadden Sea. J. Exp. Mar. Biol. Ecol. 89: 97-107.
Kinne, O. 1971. Salinity: Invertebrates. In O. Kinne (ed.). Marine Ecology. Wiley-Interscience, Nueva York, EEUU.

Lara-Domínguez, A.L., A. Yáñez-Arancibia \& F.L. Amescua. 1981. Biología y ecología del bagre Arius melanopus Günther en la Laguna de Términos, sur del Golfo de México (Pisces: Ariidae). An. Inst. Cienc. Mar y Limnol. UNAM 8: 267-304.

Levins, R. 1968. Evolution in changing environments. Princeton, Nueva Jersey, EEUU.

Licona, G. \& C. Moreno. 1996. Hábitos alimenticios de peces asociados a fanerógamas acúaticas. Laguna de Términos, Campeche. Tesis Profesional Biólogo, UNAM, México D.F., México.

Mc Roy, C.P. \& C. Helfferich. 1977. Seagrass community dynamics in a subtropical lagoon. Aquaculture 12: 253-277.

Pauly, D. 1975. On the ecology of a small West- African lagoon. Sonderdruck aus Bd. 24: 46-62.

Peterson, C.H. 1982. The importance of predation and intra-specific competition in the population biology of two infaunal suspension feeding bivalves Protothaca staminea and Chione undatella. Ecological Monographies 52: 437-475.

Pinkas, I., M.S. Oliphant \& I.L.K. Iverson. 1971. Food habits of albacore, blue fin tuna and bonito in California waters. California Departament of Fish and Game, Fish. Bull.152: 1-105.

Prejs, A. \& G. Colomine. 1981. Métodos para el Estudio de los Alimentos y las Relaciones Tróficas de los Peces. Universidad Central de Venezuela y Universidad de Varsovia, Caracas, Venezuela.

Raz-Guzmán, A. 1995. Caracterización trófica de los componentes dominantes de las comunidades bentónicas en Laguna de Términos, Campeche y en la plataforma continental adyacente. Tesis Doctorado, $\mathrm{CCH}$ Instituto de Ciencias del Mar y Limnología, UNAM, México D.F., México.

Raz-Guzmán, A. \& A. Sánchez. 1992. Registros adicionales de cangrejos braquiurus (Crustacea: Brachyura) de la Laguna de Términos, Campeche. An. Inst. Biol., UNAM, Serie Zoologica 63: 29-45.

Raz-Guzmán, A., A.M. Sánchez, L.A. Soto \& F. Álvarez. 1986. Catálogo ilustrado de cangrejos Brachyurus y Anomurus de la Laguna de Términos, Campeche 
(Crustacea: Brachyura, Anomura). An. Inst. Biol., UNAM, Serie Zoologica 57: 341-384.

Rivera, A.E. 1990. Ecología trófica de dos poblaciones de peces tropicales costeros (Polydactylus octonemus y Lutjanus synagris) del sur del Golfo de México. Tesis Profesional, Facultad de Ciencias, UNAM, México D.F., México.

Rosecchi, E. \& Y. Novaze. 1987. Comparaison de cinq indices alimentaires utilisés dans I'analyse des contenus stomacaux. Revues des Travaux de l'Institut des Peches Maritimes 49: 11-123.

Rooker, J.R. 1995. Feeding ecology of the schoolmaster snapper Lutjanus apodus (Walbaum) from southwestern Puerto Rico. Bull. Mar. Sci. 56: 881-894.

Sánchez, A. 1993. Selectividad y valor del hábitat de los estadios inmaduros del camarón rosado, Penaeus $(F)$ duorarum (Crustacea: Decápoda) en Laguna de Términos, Campeche. Tesis Doctorado, $\mathrm{CCH}$. Instituto de Ciencias del Mar y Limnología, UNAM, México D.F., México.

Sánchez, A. 1994. Feeding habits of Lutjanus apodus (Osteichthyes: Lutjanidae) in Laguna de Términos, Southwest Gulf of Mexico. Rev. Invest. Mar. 15: 125-134.

Sánchez, A.M. \& A. Raz-Guzmán. 1992. Distribution patterns of aquatic brachyuran crabs (Crustacea: Decapoda) in a tropical estuarine system: Terminos Lagoon, southern Gulf of Mexico. Abstracts of the British Ecological Society \& ASLO Symposium, Aquatic Ecology: Scale, Pattern \& Process. Cork, Ireland: 60-61.

Sánchez, A.M. \& L.A. Soto. 1982. Comportamiento anual de las postlarvas epibénticas de camarones peneidos en el sector oriental de la Laguna de Términos. Congreso Nacional de Zoología, Mazatlán, México.

Secretaría de Ecología Medio Ambiente Recursos Naturales y Pesca SEMARNAP. 2000. Sistema Nacional de Información Pesquera. Subsecretaría de Pesca. Dirección de Estadística y Registros Pesqueros. Edición junio, 2000, SEMARNAP, México DF, México.

Sierra, L.M. 1996-1997. Relaciones tróficas de los jóvenes de cinco especies de pargos (Pisces: Lutjanidae) en Cuba. Rev. Biol. Trop. 44/45: 499-506.

Vargas-Maldonado, I. \& A. Yáñez-Arancibia. 1987. Estructura de las comunidades de peces en sistemas de pastos marinos (Thalassia testudinum) de la Laguna de Términos, Campeche, México. An. Inst Cienc. Mar y Limnol.,UNAM 14: 181-196.
Vargas-Maldonado, I., A. Yáñez-Arancibia \& F.L. Amescua. 1981. Ecología y estructura de las comunidades de peces en áreas de Rhizophora mangle y Thalassia testudinum de la Isla del Carmen, Laguna de Términos, sur del Golfo de México. An. Inst. Cienc. Mar y Limnol., UNAM 8: 241-266.

Weinstein, M.P. 1985. Distributional ecology of fishes inhabiting warm-temperate and tropical estuaries: Community relationships and implications, p. 285310. In A. Yáñez-Arancibia (ed.). Fish Community Ecology in Estuaries and Coastal Lagoons: Towards an Ecosystem Integration UNAM, México D.F., México.

Yáñez-Arancibia, A. \& J.W. Day Jr. 1982. Ecological characterization of Terminos Lagoon, a tropical lagoon-estuarine system in the Southern Gulf of México. In P. Lasserre \& H. Postma (eds.). Coastal Lagoons, Oceanológica Acta, Spec. Vol. 5: 431-440.

Yáñez-Arancibia, A., A.L. Lara-Domínguez, P. SánchezGil, I. Vargas-Maldonado, P. Chavance, F.L. Amezcua, A. Aguirre-León \& S. Díaz-Ruiz. 1982. Ecosystem dinamics and nichthemeral and seasonal programming of fish community structure in a tropical estuarine inlet, México. In P. Lasserre \& $\mathrm{H}$. Postma (eds.). Coastal Lagoons Oceanológica Acta Spec. Vol. 5: 417-429.

Yáñez-Arancibia, A. \& A.L. Lara-Domínguez. 1983. Dinámica ambiental de la Boca de Estero Pargo y estructura de sus comunidades de peces en cambios estacionales y ciclos de 24-hrs. (Laguna de Término s, sur del Golfo de México). An. Inst. Cienc. Mar y Limnol.,UNAM 10: 85-116.

Yáñez-Arancibia, A., A.L. Lara-Domínguez, P. Chavance \& D. Flores. 1983. Environmental behaviour of Terminos Lagoon ecological system, Campeche, Mexico. An. Inst. Cienc. Mar y Limnol., UNAM 10: 137-176.

Yáñez-Arancibia, A., A.L. Lara-Domínguez, A. AguirreLeón, S. Díaz-Ruiz, F.L. Amezcua, D. Flores \& P. Chavance. 1985a. Ecología de poblaciones de peces dominantes en estuarios tropicales: factores ambientales que regulan las estrategias biológicas y la reproducción. In A. Yáñez-Arancibia (ed.). Fish Community Ecology in Estuaries and Coastal Lagoons: Towards an Ecosystem Integration, UNAM, México D.F., México 15: 311-366.

Yáñez-Arancibia, A., A.L. Lara-Domínguez, P. SánchezGil, M.C. García-Abad, H. Alvarez-Guillén, M. Tapia-García, D. Flores \& F.L. Amescua. 1985b. Ecology and evaluation on fish community in coastal 
ecosystems: Estuary-shelf interrelationships in the southern Gulf of Mexico. In A. Yáñez-Arancibia (ed.). Fish Community Ecology in Estuaries and Coastal Lagoons: Towards an Ecosystem Integration, UNAM, México D.F., México 22: 475-498.

Yáñez-Arancibia, A. \& P. Sánchez-Gil. 1986. Los Peces Demersales de la Plataforma Continental del Sur del Golfo de México. Vol. 1: Caracterización del
Ecosistema y Ecología de las Especies, Poblaciones y Comunidades. Instituto de Ciencias del Mar y Limnología, UNAM. Publicación Especial 9. 230 p.

Zárate, L.D.J. 1996. El manglar como hábitat crítico para las comunidades nectónicas en Estero Pargo, Laguna de Términos, México. Tesis Maestría, CCH Instituto de Ciencias del Mar y Limnología, UNAM, México D.F., México. 